\title{
Formation of Rydberg states in collisions of fast hydrogen atoms with $\mathrm{H}_{2}, \mathrm{~N}_{2}$ and $\mathrm{He}$
}

\author{
S A Aseyev, Yu A Kudryavtsev and V V Petrunin \\ Institute of Spectroscopy, USSR Academy of Sciences, 142092 Troitzk, Moscow Region, \\ USSR \\ Received 16 October 1991
}

\begin{abstract}
The cross sections for production of hydrogen Rydberg states $(n=24-27)$ in collisions of fast (accelerated to $3.6 \mathrm{keV}$ ) hydrogen atoms with $\mathrm{H}_{2}, \mathrm{~N}_{2}$ and $\mathrm{He}$ were measured. Field ionization was used to select states in terms of the principal quantum number $n$. The field ionizer was calibrated by the ionization of Rydberg states selectively excited by dye lasers. It has been found that the cross sections scale with $n$ in the same way as the cross sections of hydrogen Rydberg state production, when the fast protons exchange their charge on potassium.
\end{abstract}

The excitation of hydrogen atoms to Rydberg states in collisions with other neutral atoms and molecules is of interest for experimental physics (Koch 1983) as well as in studying and simulating some processes in atmosphere and space. Also, experimental data obtained for the cross sections of this process would be useful for testing the methods being developed now in atomic collision theory (McLaughlin and Bell 1989, Borodin 1986), especially in the range of energies below $100 \mathrm{keV}$ where the Born approximation cannot be applied. However, as far as we know, there are just a few experimental works (Gostev et al 1982, Kurskov and Khakhaev 1989, Kudryavtsev and Petrunin 1991) concerned with the formation of highly excited states in collisions of neutral atoms. In all these experiments the excitation of He atoms was studied, and there are no results for hydrogen.

In this work we measured the cross sections for the formation of hydrogen Rydberg states in collisions between fast hydrogen atoms and the molecules $\mathrm{H}_{2}, \mathrm{~N}_{2}$ and $\mathrm{He}$. The experiments were performed with a set-up designed for collinear photoionization detection of rare isotopes in an accelerated atomic beam (Aseev et al 1991). The collisional excitation of Rydberg states in a fast atomic beam is one of the processes that produces a background signal limiting the detection of rare isotopes (Kudryavtsev and Petrunin 1988, Kudryavtsev et al 1988). In this work we measured the cross sections for the collisional formation of hydrogen atoms in Rydberg states using the dependence of the background signal on the gas pressure in the vacuum system. This technique was used by Kudryavtsev and Petrunin (1991) to measure the cross section for the formation of $\mathrm{He}$ Rydberg states in collisions of fast metastable $\mathrm{He}$ atoms with $\mathrm{He}, \mathrm{Ne}$ and $\mathrm{N}_{2}$.

The experimental set-up is schematically shown in figure 1. A proton beam of energy $3.6 \mathrm{keV}$ was produced by a high-frequency discharge ion source, focused and then deflected by a magnetic mass filter to free it from the molecular and neutral 


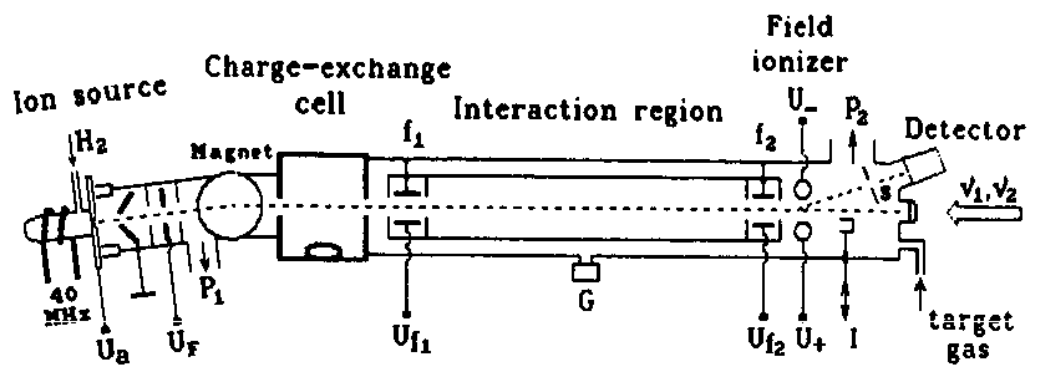

Figure 1. The scheme of the experimental set-up: I, atomic flow meter; G, pressure gauge; $f_{1}, f_{2}$, filtering capacitors; $P_{1}-P_{2}$, vacuum evacuation; $s$, variable width slit.

components of the beam. Then, in the charge-exchange cell the fast protons were recharged in potassium vapour to neutral atoms, one fourth of the atoms were produced in the metastable $2 \mathrm{~S}$ state. The unrecharged ions were removed from the neutral beam with a filtering capacitor $f_{1}$. In the electric field of this capacitor the $2 S$ and $2 P$ states of hydrogen are mixed which leads to a decay of the metastable states.

Laser excitation of Rydberg states was used in this work just to calibrate the field ionizer. When the electric field strength in the filtering capacitor $f_{1}$ was $250 \mathrm{~V} \mathrm{~cm}^{-1}$, about half the metastable atoms had time to pass through the field without decay, but all the protons were removed from the beam. After the capacitor the atomic beam entered a $110 \mathrm{~cm}$ long interaction region where two-step selective laser excitation of metastable hydrogen atoms to different Rydberg states via the intermediate $3 \mathrm{P}$ level could be realized. For this purpose, the radiation of two pulsed dye lasers $\left(\lambda_{1}=657 \mathrm{~nm}\right.$, $\lambda_{2}=830 \mathrm{~nm}$ ) pumped by a copper-vapour laser was directed collinearly with the atomic beam. When entering the electric field of two ionizing cylinders the Rydberg atoms were ionized, and the resulting ions were deflected by the same field into the variablewidth slit in front of the detector.

Such an ionization scheme is characterized by dispersion with respect to $n$, since different Rydberg states are ionized at different electric field strength and, hence, are subjected to the deflecting action of the field to different degrees.

Figure 2( $a$ ) shows how the photoion signals depend on the voltages of the ionizing cylinders at laser excitation of Rydberg states with $n=23$ and $n=27$. The peaks of the adjacent Rydberg states are considerably overlapped due to the diabatic character of ionization of atoms in an electric field typical of hydrogen. The peak observed at voltages $U_{+}=-U_{-}=910 \mathrm{~V}$ is conditioned by the direct photoionization of metastable atoms to continuum as two quanta of first-step laser radiation $\lambda_{1}$ are absorbed. The photoion signal disappeared completely at high field strength in the capacitor $f_{2}$ $\left(U_{\mathrm{f}_{2}}>3 \mathrm{kV}\right)$ which ionized all the Rydberg atoms before they entered the field of the ionizing cylinders.

The atomic beam current was about $10^{10}$ atoms per second and was measured with the use of a detector based on secondary electron emission. The secondary electron emission coefficient for hydrogen atoms was assumed to be 1.18 times higher than for protons (Thomas 1985). The coefficient of secondary electron emission from the tantalum bottom of the detector for protons measured in the case of a cold chargeexchange cell and with switched off voltages $U_{\mathrm{f}_{1}}, U_{\mathrm{f}_{2}}, U_{+}$and $U_{-}$was $1.02 \pm 0.05$. the ion current in this case was measured with a Faraday cup. The detection efficiency of the secondary electron multiplier was measured by deflecting the ion beam into the detector by means of ionizing cylinders $\left(U_{+}=-U_{-}=910 \mathrm{~V}\right)$. It was equal to $0.39 \pm 0.05$. 


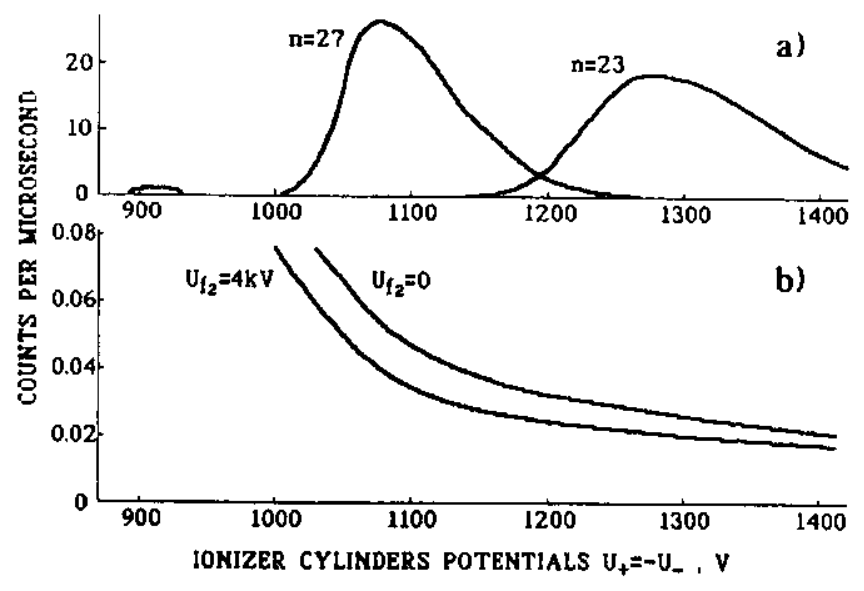

Figure 2. (a) The photoion signal as a function of the voltages of the ionizer cylinders $U_{+}=-U_{-}$at laser excitation of Rydberg states with $n=23$ and $n=27$. (b) The ion signal as a function of the voltages of the ionizer cylinders $U_{+}=-U_{-}$with switched off and on voltage on the filtering capacitor $f_{2}$. Atomic flow $1.1 \times 10^{10} \mathrm{~s}^{-1}$, hydrogen pressure $1.3 \times$ $10^{-5}$ Torr, slit width $2 \mathrm{~mm}$.

When we studied collisional processes, the field strength in the filtering capacitor $f_{1}$ was $9 \mathrm{kV} \mathrm{cm}^{-1}$. All the metastable $2 \mathrm{~S}$ states in this case decayed completely, and all the highly excited states with $n>16$ which could be formed in the charge exchange cell were ionized.

As the atomic beam propagated in the interaction region and collided with the molecules of the gas let into the system, we could observe both collisional excitation of Rydberg states and collisional ionization of fast hydrogen atoms. With the voltage of the ionizing cylinders $U_{+}=-U_{-}=910 \mathrm{~V}$, when all the ions from the interaction region reached the detector, we determined the collisional ionization cross section of hydrogen atoms $\sigma_{01}$ for different gases from the pressure dependence of the ion signal. The values obtained are given in table 1. The results of our measurements are in good agreement with most of the data for $\sigma_{01}$ available in the literature (Tawara 1978, Nakai et al 1987) which points to the absence of large experimental errors.

Figure $2(b)$ shows the ion signal as a function of the voltages of the ionizing cylinders with the voltage of the filtering capacitor $f_{2}$ switched on and off and with the hydrogen pressure in the vacuum system being $1.3 \times 10^{-5}$ Torr. The part of the ion signal conserved at high voltages $U_{\mathrm{f}_{2}}$ was conditioned by the collisional ionization of fast atoms directly in the region of the electric field of the ionizing cylinders. The other

Table 1. Experimental cross sections of the ionization $\left(\sigma_{0 t}\right)$ and of the excitation to the Rydberg state with $n=24\left(\sigma_{00}^{24}\right)$ of hydrogen atoms accelerated to $3.6 \mathrm{keV}$ in collisions with $\mathrm{H}_{2}, \mathrm{~N}_{2}$ and $\mathrm{He}$.

\begin{tabular}{lcl}
\hline $\begin{array}{l}\text { Target } \\
\text { gas }\end{array}$ & $\sigma_{01}\left(10^{17} \mathrm{~cm}^{2}\right)$ & $\sigma_{00}^{24}\left(10^{20} \mathrm{~cm}^{2}\right)$ \\
\hline $\mathrm{H}_{2}$ & 7.4 & 2.4 \\
$\mathrm{~N}_{2}$ & 16.1 & 2.6 \\
$\mathrm{He}$ & 8.3 & 1.4 \\
\hline
\end{tabular}


part of the signal that disappeared as $U_{\mathrm{f}_{2}}$ increased, in the same way as the laser photoion signal, was caused by the field ionization of the Rydberg atoms formed as a result of collisions in the interaction region. More detailed confirmation of such an interpretation can be found in the work by Kudryavtsev and Petrunin (1991).

The Rydberg atoms excited by collisions were subjected to diabatic ionization in the electric field similarly to laser-excited Rydberg atoms. The cross sections for the formation of Rydberg states were determined from the dependence of the signal on gas pressure as is described by Kudryavtsev and Petrunin (1991). The excitation cross sections for states with $n=24$ are listed in table 1. The absolute error of these measurements is estimated as $+80 /-45 \%$. The relative error for various gases does not exceed $20 \%$. It should be noted that the obtained values of collisional excitation cross sections $\sigma_{00}^{n}$ are much larger than these in proton charge exchange $\sigma_{10}^{n}$, with the energies and the target gases being the same (King and Latimer 1979).

With increasing $n$ the cross section $\sigma_{00}^{n}$ decreases. Assuming that this decrease follows the scaling law $\sigma_{00}^{n} \sim n^{-\alpha}, \alpha$ is found to be $3 \pm 1.2$. The large error in the determination of $\alpha$ is conditioned by the small range of $n$ and by the considerable relative error in the measurement $\sigma_{00}^{n}$ for different $n$. However, a systematic error can be excluded if we compare the $n$ distribution of the Rydberg atoms formed in collisions of neutral atoms and in proton charge exchange. To observe the Rydberg atoms formed by proton charge exchange on potassium the field in $f_{1}$ was decreased to $250 \mathrm{~V} \mathrm{~cm}^{-1}$ $\left(U_{\mathrm{f} 2}=0\right)$. The highly excited atoms with $n<30$ passed through the filtering capacitors and were ionized in the electric field of the cylinders.

At low temperature of the charge exchange cell, when less than $40 \%$ of the ion beam was recharged, the ratio of the flow of atoms with $n=24$ to the total flow of recharged atoms $I_{24} / I$ was independent of temperature and determined by the ratio $\sigma_{i 0}^{24} / \Sigma_{n} \sigma_{i 0}^{n}$, where $\sigma_{i 0}^{24}$ is the cross section of proton charge exchange to a state with $n=24$ and $\Sigma_{n} \sigma_{10}^{n}$ is the total cross section of proton charge exchange. The value $I_{24} / I=2.8 \times 10^{-5}$ is in agreement with the results obtained by McFarland and Futch (1970).

The dependences of the 'Rydberg' part of the signal (vanishing with an increase in $U_{\mathrm{f} 2}$ ) on the voltage of the ionizing cylinders in the case of proton charge exchange and in the case of collisional excitation of fast atoms were found to be identical. If we assume that the scaling law $\sigma_{10}^{n} \sim n^{-3}$ is fulfilled in proton charge exchange, we can get for collisional excitation, with allowance for our experimental errors, $\sigma_{00}^{n} \sim n^{-\alpha}$, where $\alpha=3 \pm 0.4$. For a proton beam energy $\geqslant 4 \mathrm{keV}$ the fulfilment of the scaling law $\sigma_{10}^{n} \sim n^{-3}$ is confirmed experimentally by many authors (see the review by Koch 1983). But, as far as we know, in collisions of neutral particles this scaling rule has not been demonstrated before.

\section{References}

Aseev S A, Kudryavtsev Yu A, Letokhov V S and Petrunin V V 1991 Opt. Lett. 16 514-6

Borodin V M 1986 Voprosy Teorii Atomnykh Stolknovenii vol 3 (Leningrad: Leningrad University Press) pp 72-82 (in Russian)

Gostev V A, Elakhovsky D V and Khakhaev A D 1982 Opt. Spektrosk. 52 909-10 (Engl. transl. Opt. Spectrosc. 52 544-5)

King R F and Latimer C J 1979 J. Phys. B: At. Mol. Phys. 12 1477-84

Koch P M 1983 Rydberg States of Atoms and Molecules (New York: Cambridge University Press) pp 473-512

Kudryavtsev Yu A, Letokhov V S and Petrunin V V 1988 Opt. Commun. 68 25-30 
Kudryavtsev Yu A and and Petrunin V V 1988 Zh. Eksp. Teor. Fiz. 94 76-88 (Engl. transl. Sov. Phys.-JETP 67 691-7)

- 1991 Zh. Eksp. Teor. Fiz. 99 81-92 (Engl. transl. Sov. Phys.-JETP 72 43-9)

Kurskov S Yu and Khakhaev A D 1989 Izv. Akad. Nauk. SSSR. Ser. Fiz. 53 1689-96

McFarland R H and Futch A H 1979 Phys. Rev. A 2 1795-801

McLaughlin B M and Bell K L 1989 J. Phys. B: At. Mol. Opt. Phys. 22 763-76

Nakai Y, Shirai T, Tawara H and Ito R 1987 At. Data. Nucl. Data Tables 37 69-102

Tawara H 1978 At. Data Nucl. Data Tables 22 491-525

Thomas E W 1985 Particle Interaction with Surfaces (Oak Ridge, TN: Oak Ridge National Laboratory) ORNL-6088 pp C34-5 